\title{
Modelling of Vehicles with Integrated Antennas
}

\author{
J.C. Batchelor ${ }^{1}$, R.J. Langley ${ }^{1}$, H. Endo ${ }^{2}$ and M. Saito ${ }^{2}$
}

This is the peer reviewed version of the following article (J.C. Batchelor, R.J. Langley,

H. Endo and M. Saito, MODELLING OF VEHICLES WITH INTEGRATED ANTENNAS, Wiley, Microwave and Optical Technology Letters, 27, (6), December 2010, pp. 404-407), which has been published in final form at (http://onlinelibrary.wiley.com/doi/10.1002/1098-2760(20001220)27:6\%3C404::AIDMOP11\%3E3.0.CO;2-2/abstract). This article may be used for non-commercial purposes in accordance with Wiley Terms and Conditions for self-archiving 


\title{
Modelling of Vehicles with Integrated Antennas
}

\author{
J.C. Batchelor ${ }^{1}$, R.J. Langley ${ }^{1}$, H. Endo ${ }^{2}$ and M. Saito ${ }^{2}$ \\ ${ }^{1}$ Electronics Laboratory, The University of Kent, Canterbury, Kent, CT2 \\ $7 \mathrm{NT}$. \\ ${ }^{2}$ Harada European Technology Centre, Research and Development \\ Building, University of Kent, Canterbury, Kent, CT2 7PD.
}

\section{Corresponding Author:}

j.c.batchelor@ukc.ac.uk

Fax: +44 1227456084

\begin{abstract}
Modelled results are presented for an on-glass car antenna designed primarily for reception at VHF. A discussion is given as to the electromagnetic significance of various parts of the car structure with respect to received pattern shape and computational efficiency. The simulated results are verified by comparison with measurement.
\end{abstract}

Key words: numerical modelling, on-glass antennas, printed antennas, vehicular FM antennas, $N E C-2$

\section{Introduction}

Recent developments in automobile design no longer favour the traditional fender mounted whip type antenna. The reasons being firstly aesthetic, and secondly, the rapidly escalating number and type of mobile communication facilities available. The corresponding broad spectrum and range of radiation patterns necessary to encompass these new services require more than one antenna for adequate transmission. It is therefore desirable to integrate a suite of conformal antennas invisibly on 
the vehicle. Many recent studies of antenna behaviour on automobiles have involved mobile systems at UHF and beyond. However, modelling at VHF is also an important consideration as the car body is resonant at these frequencies. Also the continued use of analogue transmission requires optimal antenna performance in order to maximise the quality of the received signal. In this work the conducting car body is modelled using version 2 of the NEC code which allows wire mesh models to be implemented. This paper will briefly discuss some of the important issues involved in modelling printed antennas mounted on car rear windscreens. The wire grid nature of NEC means that feed points are not well represented and so input impedances are not accurately simulated, therefore emphasis will be placed on radiation pattern modelling. Finally some modelled results will be compared with measurements taken at the Harada Industries test facility in Japan.

\section{Structure modelling}

It is generally accepted when creating representations of objects for electromagnetic simulation that not all structural details will affect electromagnetic field distributions equally. Reducing model complexity is a valuable process as the associated modelling time can be diminished considerably. While it is possible to produce elaborate and exact dimensional representations of car bodies [1], the object of this work is to investigate how the structure may be simplified for faster modelling throughput. A wire mesh model of the Nissan Gloria car is shown in Fig.1. Version 2 of the NEC code is a moment method wire segment simulator that does not model dielectric materials and it was not possible therefore to include the glass windscreens. This omission has been justified in previous work as the electrical thickness of the glass at FM frequencies (92 MHz centre) is very small and its effect is not expected to 
be significant [2]. A finite electrical parameter ground beneath the car was included though the absolute values of permittivity, $\varepsilon_{\mathrm{r}}$, and conductivity, $\sigma$, were not critical. Values of $\varepsilon_{\mathrm{r}}=5$ and $\sigma=0.001 \mathrm{Sm}^{-1}$ corresponding to urban areas were generally used. It was also found that the image modified Fresnel Reflection Coefficient ground gave results comparable to those of the more complex Sommerfeld-Norton model which is usually deemed necessary where structures are located within about $0.1 \lambda$ of the ground. The use of the simpler Fresnel Reflection Coefficient ground offered a considerable saving in simulation time.

\section{Car wire mesh}

The car body was initially represented as a rectangular box with the door supports (B-pillars) mounted vertically midway along the cabin. Most automobiles are around $1.5 \mathrm{~m}$ wide which gives a half wavelength resonance at $98 \mathrm{MHz}$. It is important, therefore, that the width to length aspect ratio of the car body is correct as it essentially acts as a leaky cavity with a fundamental resonance within the FM band. The effect of altering car length, keeping width constant, is shown in Fig.2. When the total length was $4.4 \mathrm{~m}$ (2.6 times the width) $10 \mathrm{~dB}$ nulls at $\pm 150^{\circ}$ and deeper than $20 \mathrm{~dB}$ nulls at $\pm 60^{\circ}$ in the V-polarisation are observed. The rear nulls are filled in as the car is reduced in the $\mathrm{x}$-dimension and the $\mathrm{V}$ polarised gain becomes less directional. The nulls at $\pm 60^{\circ}$ are filled by up to $11 \mathrm{~dB}$ as the car length is reduced by $20 \%$. The antenna is mounted on the rear windscreen, and the dependence of the rear V-polarised gain on the cabin length illustrates how strongly coupled the antenna performance is to the car body. Cancellation due to the halfwave cavity effect causes characteristic nulls in the vertical polarised fields at boresight directions to the car body. As vehicle mounted antennas often suffer nulls at angles 
close to $\pm 60^{\circ}$, it is a useful insight to know that they are influenced by the length of the vehicle. The H-polarised gain is affected much less by car length, the most notable change being a broadening in the forward null as length is decreased.

As the antennas considered in this work are for use in terrestrial broadcast systems, only radiation patterns at low elevation angles were considered, and pattern correlation to measurement was found to be relatively insensitive to exact roof height when compared to width/length ratio. All patterns were simulated at an elevation of $2^{\circ}$ to correspond with the receiver height of the test range.

The next most significant part of the car structure was the front supports or A-pillars. The fields scattered by these front wires interact with the door supports (B-pillars). It was observed that, although the roof supports could be modelled with single wires, their positions were important to within about $3 \%$ of a wavelength and the slope of the Apillars had to be correct to ensure correct coupling between the vertical and horizontal polarized fields. If the A-pillar elevation is too low then nulls of more than $5 \mathrm{~dB}$ arise in the $\mathrm{H}$-polarised patterns at $\phi= \pm 80^{\circ}$ which correspond to an increase in the V-polarised fields at the same angles.

Wires are modelled within NEC using a small radius kernel, though it is possible to represent wires with significant surface areas by using the extended radius feature. Increasing the radii of the A and B-pillars to give surface areas approximately equal to their physical areas made a significant improvement to the correlation between measured and predicted patterns. Roof supports with insufficient surface area cause the 
V-polarised patterns to be low in gain by $3 \mathrm{~dB}$ in the rear direction, while the H-polarised gain suffers nulls at $\phi= \pm 65^{\circ}$ which are $3.5 \mathrm{~dB}$ below the correct level.

\section{Rear screen slope}

The ratio of horizontal to vertical polarisation reception of the on-glass antenna is affected by the elevation of the screen on which the antenna is printed. The effect of changing screen slope is illustrated in Fig.3. The Hpolarisation is influenced by the rear screen elevation most strongly, the rear H-polarised gain increasing with decreasing forward gain as the screen slope is reduced. If the screen elevation is increased, the rear null deepens and becomes more central as slope of $45^{\circ}$ is reached. Steepening the screen slope also increases the side radiation slightly for both polarisations. As modern automobiles can have screen elevations considerably less than $45^{\circ}$, sometimes as low as $30^{\circ}$, a deleterious effect on the omni-directionality of an integrated antenna results.

\section{C-pillars}

The proximity of the rear roof supports or C-pillars to the on-glass antenna produces a significant influence on the radiation patterns. The width of the C-pillars affects both the Vertical and H-polarisation. Figure 4 shows how a narrower C-pillar results in a deeper H-polarised null at the rear, while the front Horizontal polarisation is also reduced slightly and nulls at $\pm 70^{\circ}$ deepen by several decibels. The main effect on the Vertical polarisation when the C-pillar is narrowed from 0.5 to $0.3 \mathrm{~m}$ is to increase the side gain by about $3 \mathrm{~dB}$. The actual C-pillar of the Nissan Gloria tapers towards the top. In the NEC-2 model however, serious numerical errors result when adjacent wire volumes overlap. This 
inaccuracy was avoided by modelling the C-pillars with parallel wires of axis separation set according to circumferential area. No significant loss in accuracy was observed.

\section{Roof}

A final important parameter of the wire model with a strong influence on correlation between simulation and measurement was the number of segments in the roof of the car. This proved to be more important than the number of segments in any other part of the model, including the boot and the rear roof supports (C-pillars) which are located immediately to the sides of the on-glass antenna. With segments $0.04 \lambda$ in length across the roof, the $\mathrm{V}$ and H-polarised patterns were both of the correct shape but were $3 \mathrm{~dB}$ below the correct levels. When the number of wires along the roof was increased by $20 \%$, which entailed reducing the segment length across the roof to around $0.03 \lambda$, the simulated gain improved significantly. The segment length along the roof was also of the order of $0.03 \lambda$.

\section{Measurement correlation}

With the above considerations taken into account it was possible to model vertical gain to within about $1 \mathrm{~dB}$ of measurement. It was more difficult to model the horizontal gain accurately, especially as the measurement contained an unexpectedly high level of asymmetry, though it was possible to predict the gain to within about $3 \mathrm{~dB}$ of its measured value around the azimuth. Although the simulated car structure was greatly simplified in many respects, the modelled radiation patterns were found to agree well with measurement. 


\section{Conclusion}

The investigation briefly described in this paper has outlined the electromagnetically important structures of a car body housing an onglass rear screen antenna. It has been indicated that the length to width aspect ratio of the body is important together with the positions of the roof supports and the slope of both the front and rear windscreens. In addition to position, the diameter of the roof supports was found to be particularly important. The width of the rear roof supports was particularly important. Overall gain levels can be matched to those of measurement by ensuring that sufficient segments are provided to grid the roof. Finally, the results are found to be generally quite insensitive to finite electrical ground parameters. It has been demonstrated that elaborate representation of vehicle bodies is not required to produce simulated radiation patterns of good accuracy. For cases where input impedance is simulated it would be necessary to model the area local to the feed in detail and also the glass dielectric effect would have to be included in the model. 


\section{References}

1 J. Shim, S.-S. Lee, J.-H. Lee, J.-G. Kang, J.-C. Koo and H.-T. Kim, Selection of the optimum feeding point of heater-grid antennas using NEC-2 moment method code, Microwave and Opt. Tech. Letts., Vol.22, (1999), 310-314.

2 R. Abou-Jaoude, and E.K.Walton, Numerical Modeling of OnGlass Conformal Automobile Antennas, IEEE Trans. Antennas Propagat. Vol.AP-46 (1998), 845-852. 
Figure Captions:

Figure 1.

Wire grid model of Nissan Gloria Car body.

(a) Side Elevation. (b) Top plan view (base wires not shown for clarity).

Figure 2:

Radiation patterns resulting from varied vehicle length. Cabin width $=$ $1.7 \mathrm{~m}$.

(a) Vertical polarisation (b) Horizontal polarisation

$$
\begin{aligned}
& \longrightarrow \text { length }=4.4 \mathrm{~m} \\
& \text { - } \text { - }- \text { - } \text { length }=4.0 \mathrm{~m} \\
& \text { - - * - } \cdot \text { length }=3.6 \mathrm{~m}
\end{aligned}
$$

Figure 3.

Radiation patterns resulting from varied rear screen slope.

$$
\begin{aligned}
& \longrightarrow \theta=40^{\circ} \text {; Vertical polarisation } \\
& \text { — - - } \theta=40^{\circ} \text {; Horizontal polarisation } \\
& \longrightarrow \quad \theta=46^{\circ} \text {; Vertical polarisation } \\
& \text { - - - - - - } \quad \theta=46^{\circ} ; \text { Horizontal polarisation }
\end{aligned}
$$

Figure 4.

Radiation patterns resulting from varied C-pillar width.
(a) Vertical polarisation
(b) Horizontal polarisation

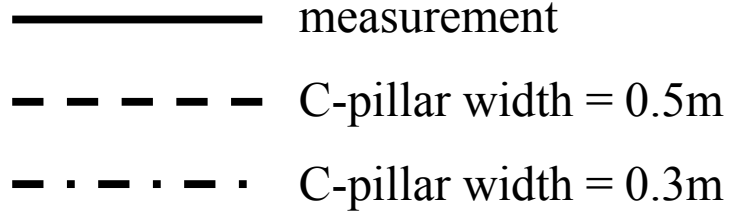


Figure 1a

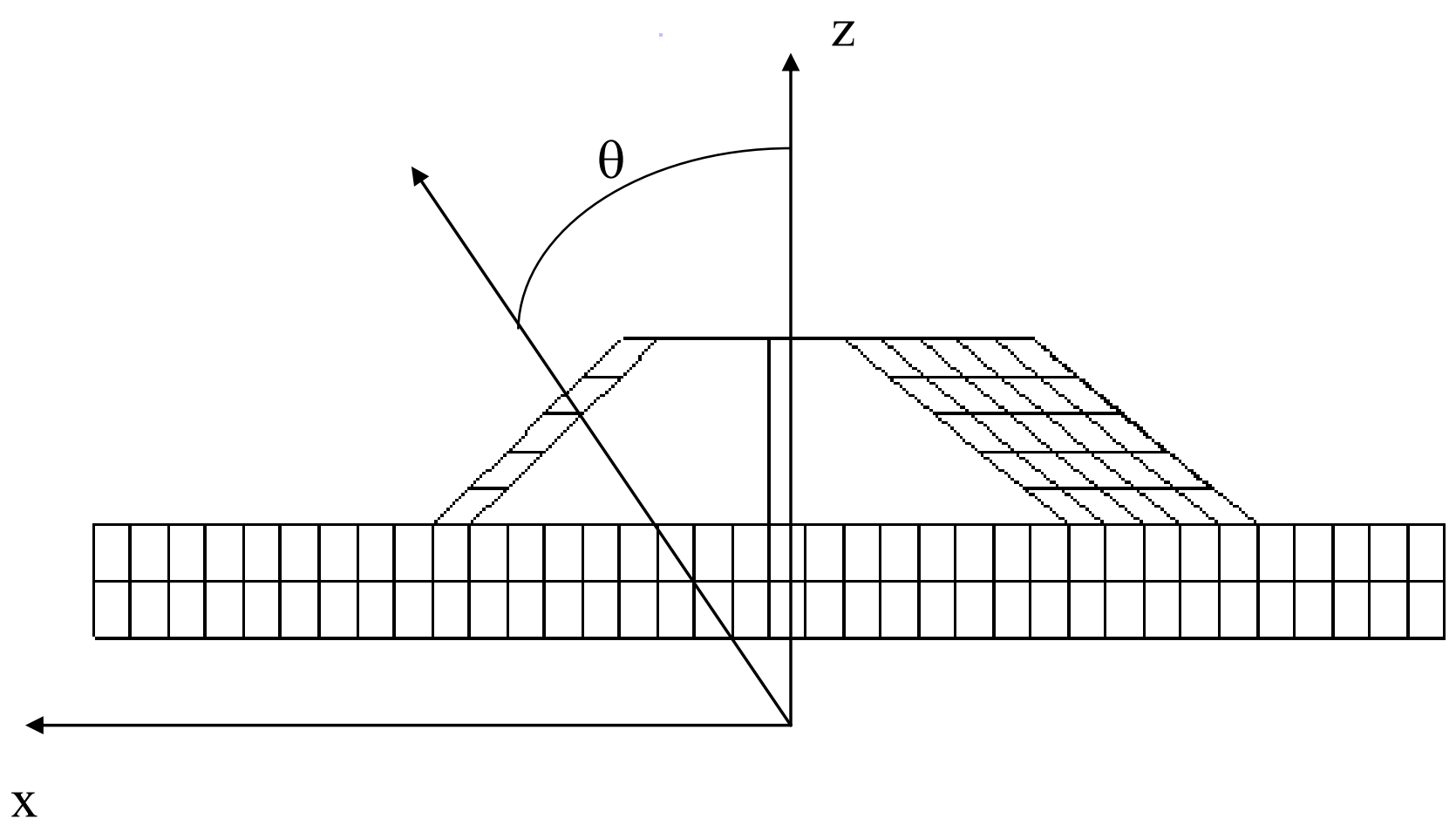


Figure $1 b$

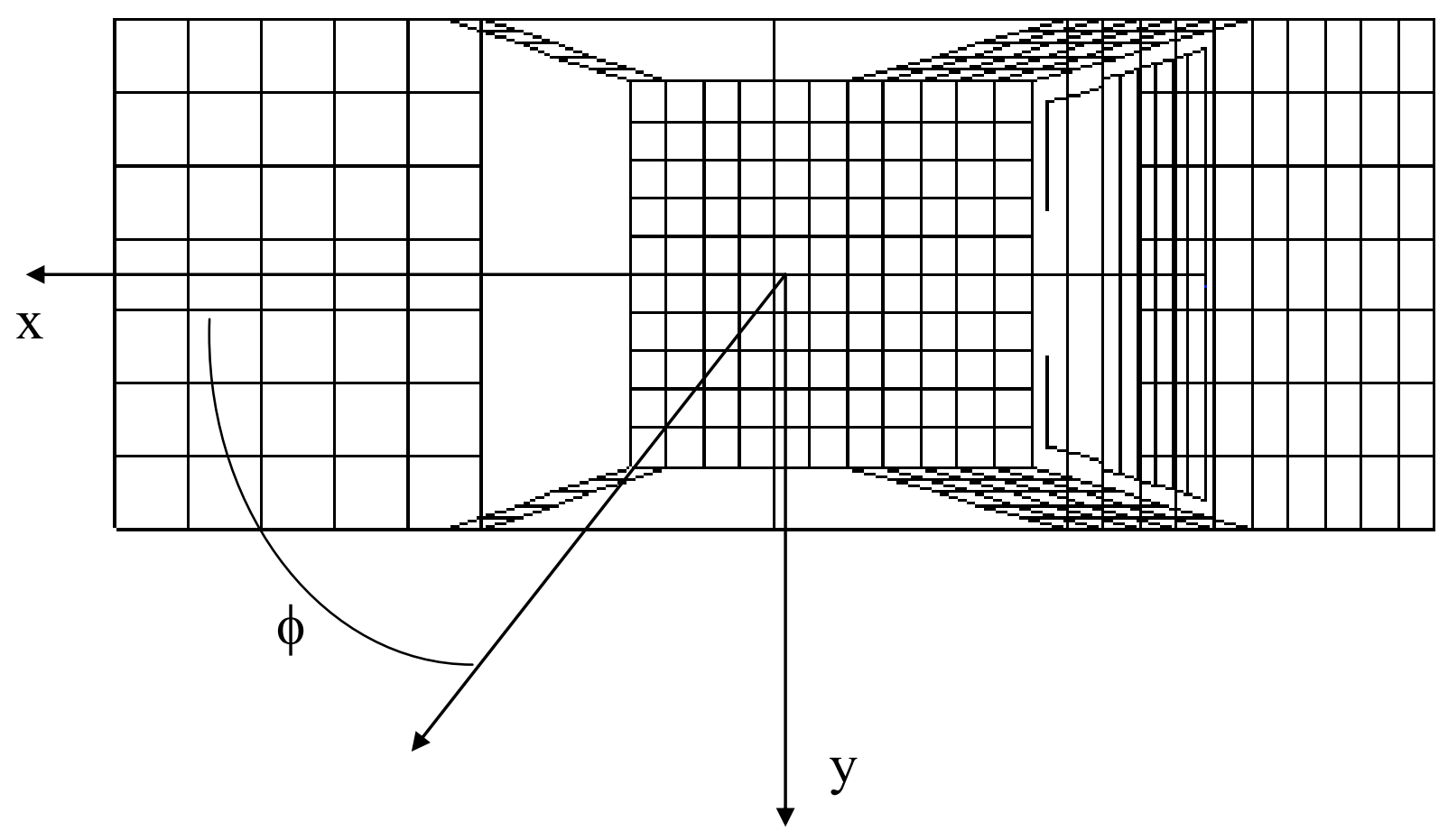


Figure 2a

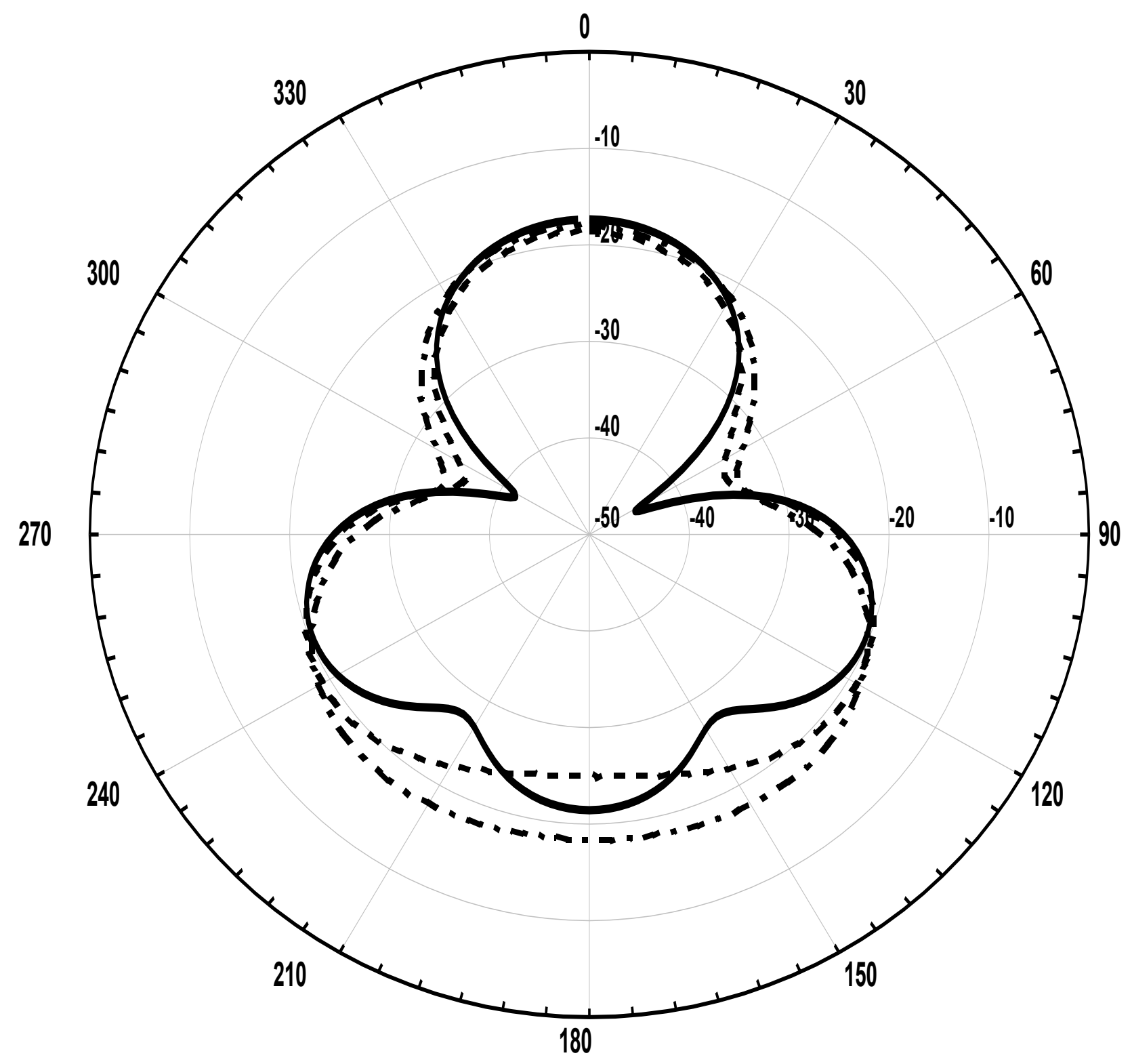


Figure $2 b$

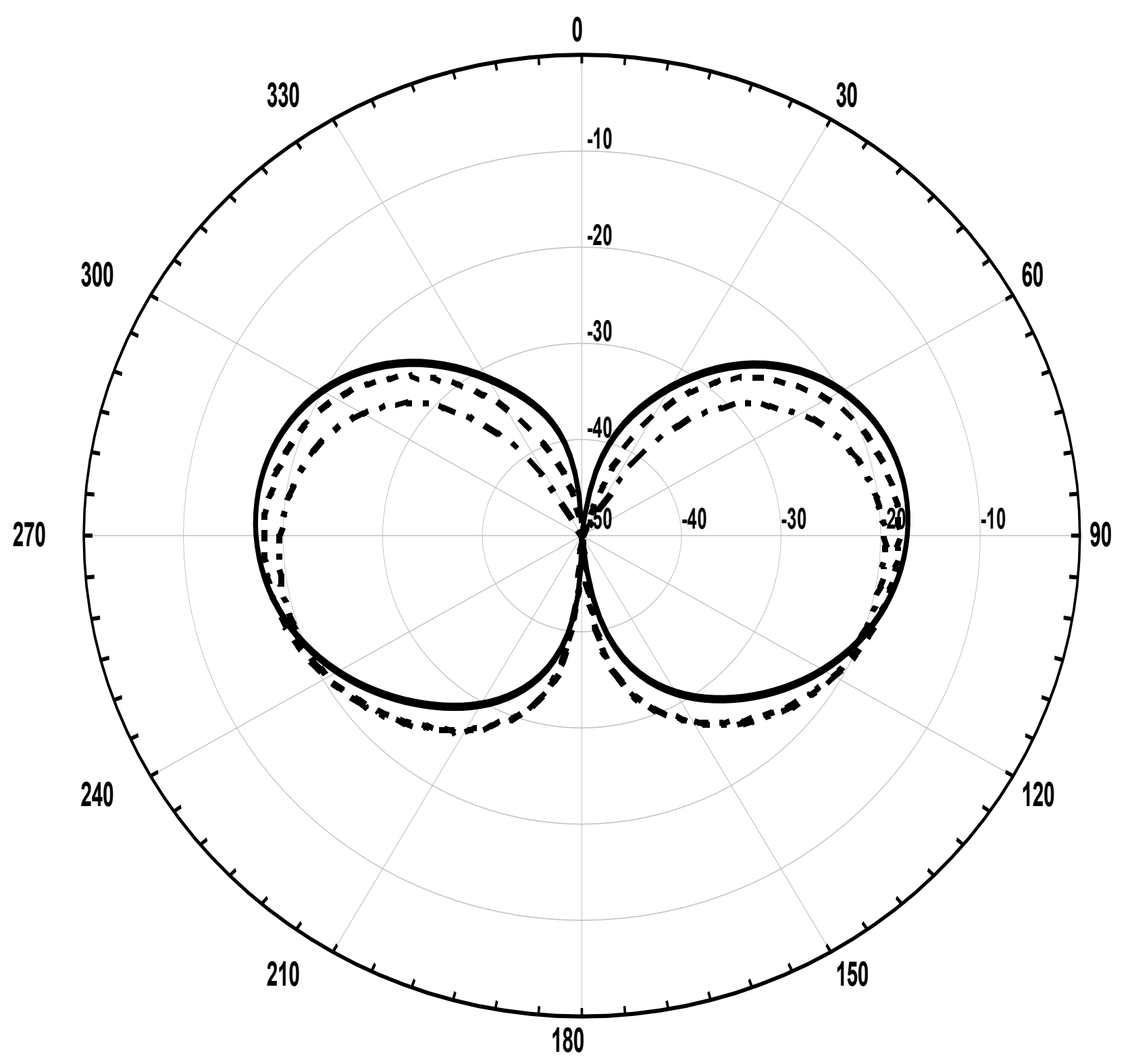


Figure 3.

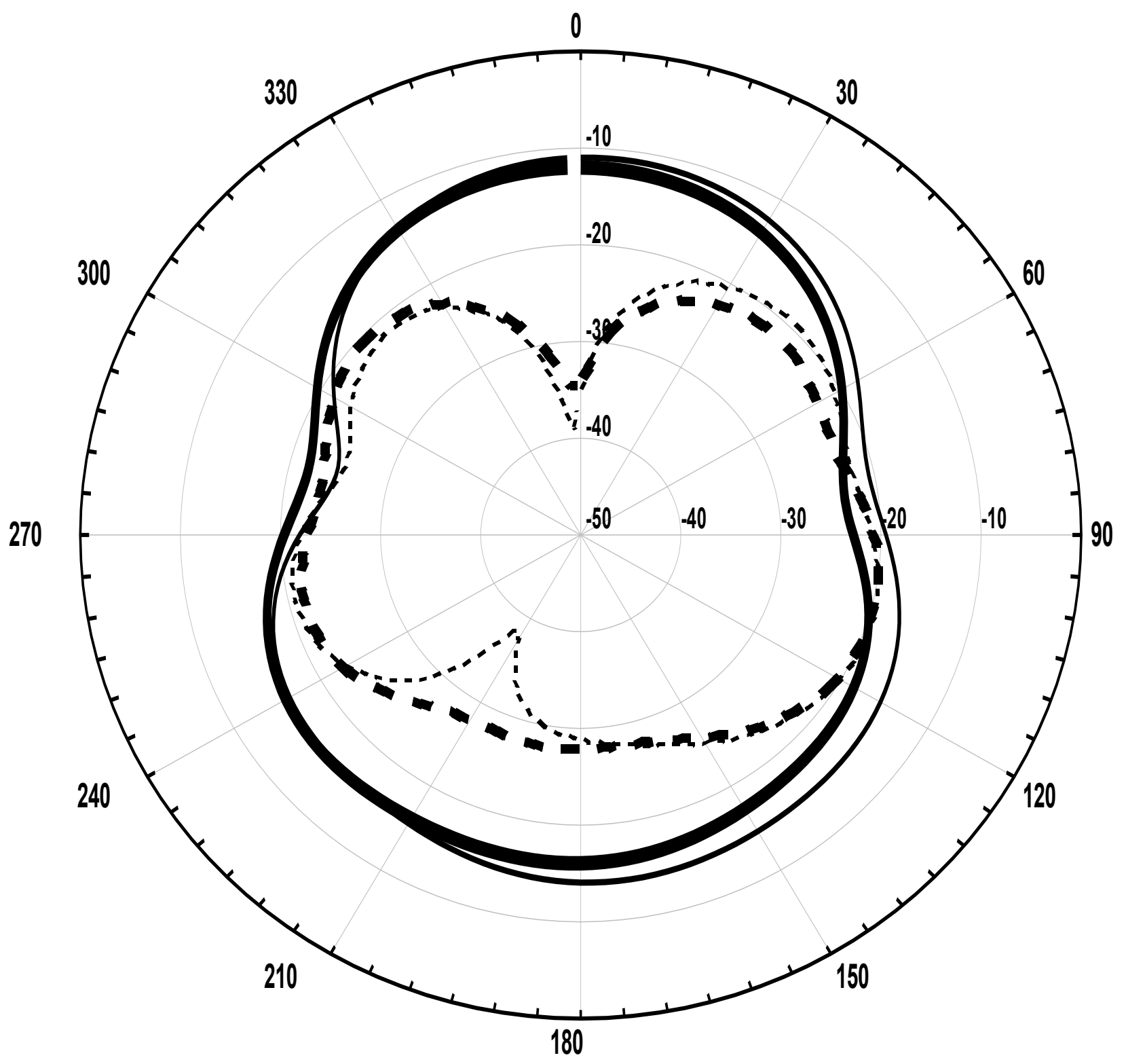


Figure 4a.

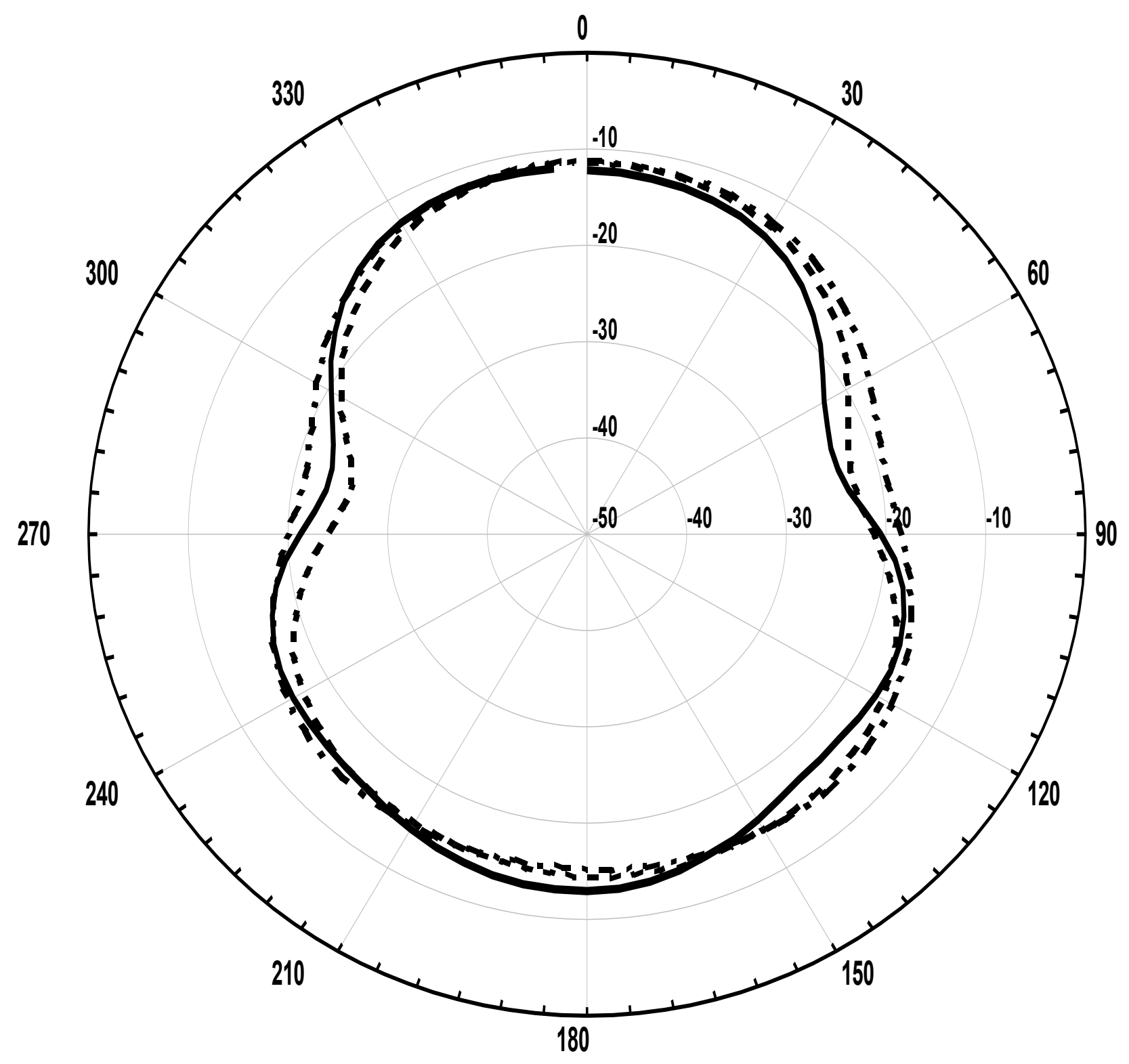


Figure $4 b$.

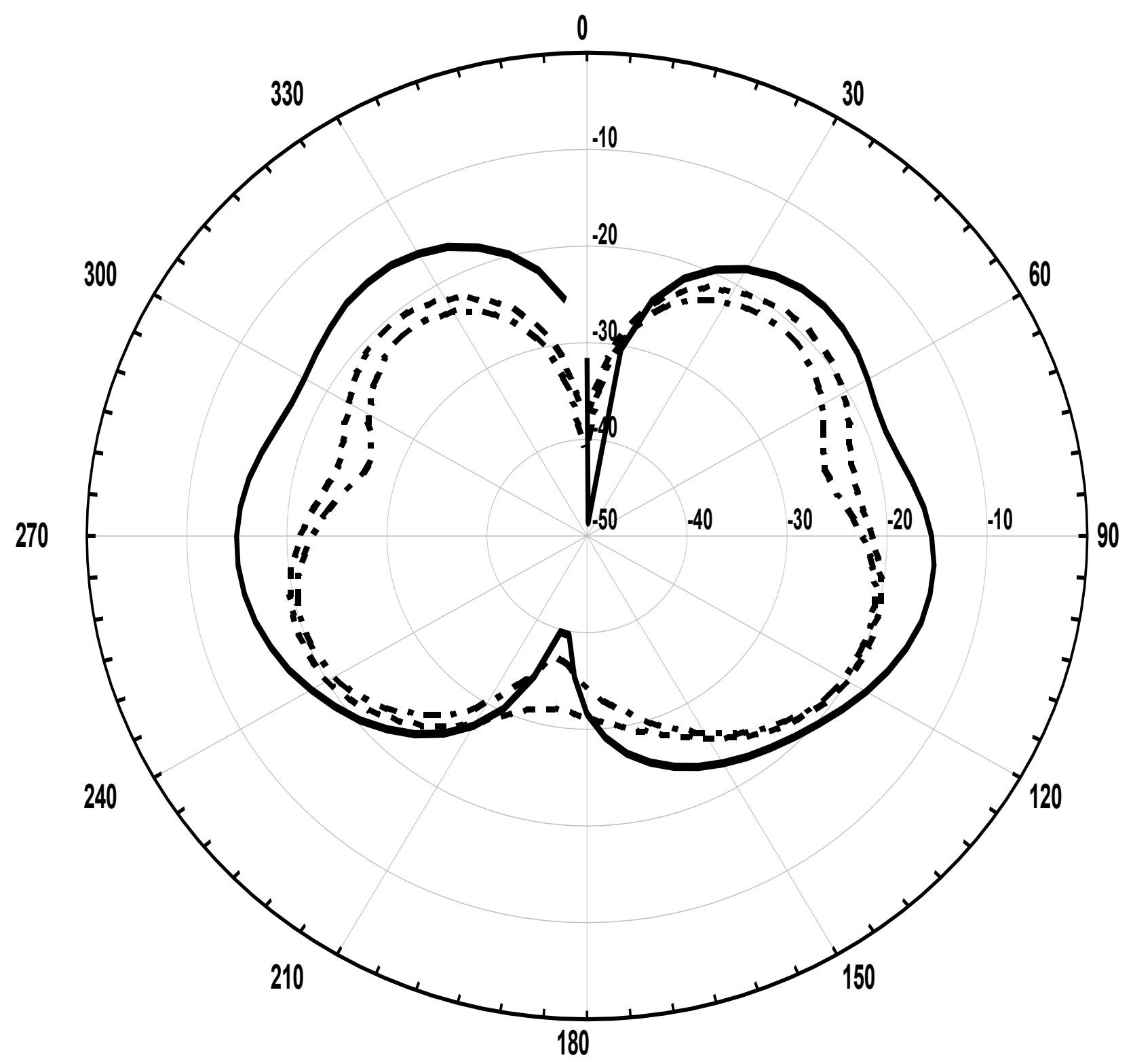

